\title{
Proof of a Conjecture of Segre and Bartocci on Monomial Hyperovals in Projective Planes
}

\author{
Fernando Hernando* \\ Department of Mathematics \\ San Diego State University \\ United States \\ Gary McGuire ${ }^{\dagger}$ \\ School of Mathematical Sciences \\ University College Dublin \\ Ireland
}

July 5, 2018

To Richard M. Wilson on the occasion of his 65 th birthday

\begin{abstract}
The existence of certain monomial hyperovals $D\left(x^{k}\right)$ in the finite Desarguesian projective plane $P G(2, q), q$ even, is related to the existence of points on certain projective plane curves $g_{k}(x, y, z)$. Segre showed that some values of $k\left(k=6\right.$ and $\left.2^{i}\right)$ give rise to hyperovals in $P G(2, q)$ for infinitely many $q$. Segre and Bartocci conjectured that these are the only values of $k$ with this property. We prove this conjecture through the absolute irreducibility of the curves $g_{k}$.
\end{abstract}

\section{Introduction}

An oval in the finite Desarguesian projective plane $P G(2, q)$ is a set of $q+1$ points with the property that no three points are collinear. If $q$ is odd then such a set is maximal with that property, and a celebrated theorem of Segre (1955) states that all such ovals are given algebraically by irreducible conics. If $q$ is

\footnotetext{
${ }^{*}$ Inspire postdoctoral researcher at the Claude Shannon Institute, funded by Irish Research Council for Science, Engineering and Technology adn Science Foundation Ireland Grant 06/MI/006, also partially supported by MEC MTM2007-64704 (Spain).

${ }^{\dagger}$ Research supported by the Claude Shannon Institute, Science Foundation Ireland Grant 06/MI/006.
} 
even however, the situation is more interesting. Here, a set of points in $P G(2, q)$ of largest possible size such that no three are collinear has cardinality $q+2$, and is called a hyperoval.

From now on in this paper we assume that $q$ is even. A hyperoval can be constructed from a (nonsingular) conic by adjoining the point at which all the tangents of the conic meet, the nucleus. Such hyperovals are generally called regular hyperovals. For $q>8$ there also exist irregular hyperovals which are not of the form conic plus nucleus, see [2, 3], 9, for example.

We represent the points of $P G(2, q)$ as homogeneous triples with coordinates from $G F(q)$. It is well known that all hyperovals can be written in the form

$$
\{(1, x, f(x)): x \in G F(q)\} \cup\{(0,0,1),(0,1,0)\}
$$

where $f(x)$ is a polynomial with certain properties, see [4, 3]. Denote the above set by $D(f(x))$. In this paper we shall examine the case where $f(x)$ is a monomial, say $f(x)=x^{k}$. If $q=2^{e}$, Segre showed that the set $D\left(x^{k}\right)$ is a hyperoval for the following values of $k$ and the values of $e$ indicated:

$$
\begin{array}{cl}
k=2^{i}, & \text { when }(i, e)=1 \quad([10,1957), \\
k=6, & \text { when }(2, e)=1([11], 1962) .
\end{array}
$$

We wish to consider other values of $k$. In particular, we wish to consider the question of whether there are other such infinite sequences, i.e. other fixed values of $k$ for which $D\left(x^{k}\right)$ is a hyperoval for infinitely many $q$. This question was previously studied by Segre and Bartocci 12 . Our main result is the following theorem, which was conjectured in [12].

Theorem 1.1. For any fixed even positive integer $k$, if $k \neq 6$ and $k \neq 2^{i}$ then the set $D\left(x^{k}\right)$ is a hyperoval in $P G(2, q)$ for at most a finite number of values of $q$.

In 8 the permutation properties of $1+x+\cdots+x^{k-1}$ on $G F(q)$ are studied. It follows from [7 (p.505) that this polynomial is a permutation polynomial if and only if $D\left(x^{k}\right)$ is a hyperoval. Hence our result sheds some light on this problem. It is now trivial to see that $k$ must be even in order for $D\left(x^{k}\right)$ to be a hyperoval, since $1+x+\cdots+x^{k-1}$ maps both 0 and 1 to 1 if $k$ is odd. So we assume $k$ is even from now on. Values of $k$ which are functions of $e$ have been studied, see 2, but we do not consider this here.

In case $D\left(x^{k}\right)$ is a hyperoval, we call it a monomial hyperoval because $f(x)$ is a monomial. Following [4, we will write $D(k)$ instead of $D\left(x^{k}\right)$.

We note some projective equivalences among these hyperovals. If $D(k)$ is a hyperoval, then so is $D(m)$ where $m=1 / k, 1-k, 1 /(1-k),(k-1) / k, k /(k-1)$, and everything is modulo $q-1$. (If $(k, q-1) \neq 1$ or $(k-1, q-1) \neq 1$ then $D(k)$ is not a hyperoval.) These hyperovals are all projectively equivalent, see [4]. 
In Section 2 we shall prove the connection between the polynomials $g_{k}(x, y, z)$ and the hyperovals $D(k)$. Section 3 contains background information about algebraic curves, and classifies the singular points of $g_{k}(x, y, z)$. Section 4 computes the possible intersection multiplicities of putative factors of $g_{k}$. We shall completely factorize $g_{k}(x, y, z)$ for $k=2^{i}$ and $k=6$ in section 5 , and there we will reprove Segre's theorems on these values. In sections 6, 7, 8, we will use Bezout's theorem to prove the main theorem in parts. The result of section 6, the case $k \equiv 2(\bmod 4)$, was already proved by Segre and Bartocci 12 .

\section{Background}

The set $D(k)$ being a hyperoval in $P G(2, q)$ is equivalent to the determinant

$$
\operatorname{det}\left(\begin{array}{ccc}
1 & 1 & 1 \\
x & y & z \\
x^{k} & y^{k} & z^{k}
\end{array}\right)
$$

being nonzero for all distinct $x, y, z \in G F(q)$. Divide the determinant by $(x+$ $y)(x+z)(y+z)$ and call the resulting polynomial $g_{k}(x, y, z)$. In other words, we define a binary polynomial $g_{k}(x, y, z)$ by

$$
g_{k}(x, y, z):=\frac{x y^{k}+y x^{k}+x z^{k}+z x^{k}+y z^{k}+z y^{k}}{(x+y)(x+z)(y+z)} .
$$

Our main theorem rests on the following, which is also used by Segre and Bartocci.

Theorem 2.1. If the polynomial $g_{k}(x, y, z)$ is absolutely irreducible over $G F(2)$, or has an absolutely irreducible factor defined over $G F(2)$, then $D(k)$ is a hyperoval in $P G(2, q)$ for only a finite number of values of $q$.

Proof. A form of the Weil bound due to W. Schmidt [13] on the number of rational points on curves over finite fields shows that one can weaken the hypothesis of nonsingularity of a curve $f(x, y)$ over $G F(q)$ to absolute irreducibility, and still obtain a bound essentially the same as that of Weil.

If the polynomial $g_{k}(x, y, z)$ of degree $k-2$ is absolutely irreducible over $G F(2)$, then applying this form of Weil's theorem shows that the number $N_{e}$ of (projective) rational points $(x, y, z)$ on $g_{k}(x, y, z)$ where $x, y, z \in G F\left(2^{e}\right)$ satisfies

$$
\left|N_{e}-2^{e}\right|<(k-3)(k-4) 2^{e / 2}+(k-2)^{2}
$$

for every $e$. Once we show that the number of such rational points where some of the coordinates are equal is at most $3 k-2$, it will follow that there are rational points over $G F\left(2^{e}\right)$ with $x, y, z$ distinct for all $e$ sufficiently large. Should it happen that $g_{k}(x, y, z)$ is not absolutely irreducible over $G F(2)$ but has an absolutely irreducible factor defined over $G F(2)$, apply the same argument to this factor. 
To this end we let $p(x, y, V)=g_{k}(x, y, x+V)$, and note that projective points $(x, y, z)$ on $g_{k}(x, y, z)$ with $x=z$ are in $1-1$ correspondence with projective points $(x, y, 0)$ on $p(x, y, V)$. A simple computation (using the fact that $k$ is even) shows that

$$
p(x, y, 0)=\frac{x^{k}+y^{k}}{(x+y)^{2}} .
$$

Again we let $q(x, W)=p(x, x+W, 0)$, and note that projective points $(x, y, z)$ on $g_{k}(x, y, z)$ with $x=z \neq y$ are in $1-1$ correspondence with affine points $(x, 1)$ on $q(x, W)$. Since $q(x, 1)=x^{k}+(x+1)^{k}$, there are at most $k-1$ projective points $(x, y, z)$ on $g_{k}(x, y, z)$ with $x=z \neq y$. A similar argument holds for points $(x, y, z)$ with $x=y \neq z$ and $y=z \neq x$. Counting the projective point $(1,1,1)$ we get that there are at most $3 k-2$ rational points $(x, y, z)$ on $g_{k}(x, y, z)$ with $x, y, z$ not all distinct.

Remark: From the form of the Weil bound in (1), we can actually compute the value of $e$, say $e_{0}$, for which $N_{e}>3 k-2$ for all $e \geq e_{0}$.

Armed with this theorem, our task now is to demonstrate the absolute irreducibility of the polynomials $g_{k}(x, y, z)$ over $G F(2)$. This is how we shall prove the results of this paper.

Bearing in mind Segre's results, $g_{k}(x, y, z)$ cannot be absolutely irreducible when $k=2^{i}$ or $k=6$.

Segre and Bartocci made the following conjecture (we are paraphrasing here).

Conjecture: $g_{k}(x, y, z)$ has an absolutely irreducible factor over $\mathbb{F}_{2}$ for every even $k$ except $k=2^{i}$ and $k=6$.

We shall prove this conjecture in this paper. By Theorem 2.1 this is enough to prove our main theorem, Theorem 1.1

\section{Singular Points}

It will be shown shortly that we are allowed to work with the affine parts of the homogeneous polynomials $f_{k}(x, y, z)$ and $g_{k}(x, y, z)$. There will be no confusion if we use the same names, and so

$$
\begin{aligned}
f_{k}(x, y) & :=x y^{k}+y x^{k}+x^{k}+y^{k}+x+y \\
g_{k}(x, y) & :=\frac{f_{k}(x, y)}{(x+y)(x+1)(y+1)}
\end{aligned}
$$

and we consider the algebraic curves defined by these polynomials over the algebraic closure of $G F(2)$. Of course, $g_{k}(x, y, z)$ is absolutely irreducible if and only if $g_{k}(x, y)$ is absolutely irreducible.

For a polynomial $h$ and a point $P=(\alpha, \beta)$, write

$$
h(x+\alpha, y+\beta)=H_{0}(x, y)+H_{1}(x, y)+H_{2}(x, y)+\cdots
$$


where each $H_{i}(x, y)$ is 0 or homogeneous of degree $i$. If $m$ is the smallest integer such that $H_{m} \neq 0$ but $H_{i}=0$ for $i<m$, then $m$ is called the multiplicity of $h$ at $P$, and is denoted by $m_{P}(h)$. In particular, $P$ is on the curve associated to $h$ if and only if $m_{P}(h) \geq 1$. Also, by definition, $P$ is a singular point of $h$ if and only if $m_{P}(h) \geq 2$. The $m$ linear factors of $H_{m}$ are the tangent lines to $h(x, y)$ at $P$. The collection of tangent lines is called the tangent cone.

The singular points can be found by equating the first partial derivatives to zero. We easily calculate ( $k$ is even)

$$
\frac{\partial f_{k}}{\partial x}(x, y)=y^{k}+1, \quad \frac{\partial f_{k}}{\partial y}(x, y)=x^{k}+1 .
$$

Hence if $P=(\alpha, \beta)$ is a singular point of $f_{k}(x, y)$, then $\alpha$ and $\beta$ are $k$-th roots of unity. Write $k=2^{i} \ell$ where $\ell$ is odd and $i \geq 1$. Then $\alpha$ and $\beta$ are $\ell$-th roots of unity, This proves

Lemma 3.1. $P=(\alpha, \beta)$ is a singular point of $f_{k}$ if and only if $\alpha^{\ell}=\beta^{\ell}=1$.

It follows that $f_{k}(x, y)$ has $\ell^{2}$ singular points. It is easy to check that there are no singular points at infinity - the three partial derivatives of $f_{k}(x, y, z)$ are $x^{k}+y^{k}, x^{k}+z^{k}, y^{k}+z^{k}$, and if these all vanish and $z=0$ then $x=y=0$ which is impossible. This proves

Lemma 3.2. $f_{k}(x, y, z)$ has no singular points at infinity.

Next we pin down the multiplicities of these singular points $P=(\alpha, \beta)$ on $f_{k}(x, y)$, and how things change for $g_{k}(x, y)$. We compute that

$$
\begin{aligned}
f_{k}(x+\alpha, y+\beta) & =\sum_{j=1}^{k}\left(\begin{array}{c}
k \\
j
\end{array}\right)\left(\alpha^{-j} x^{j} y+\beta^{-j} y^{j} x+(\beta+1) \alpha^{-j} x^{j}+(\alpha+1) \beta^{-j} y^{j}\right) \\
& =F_{0}+F_{1}(x, y)+F_{2}(x, y)+\cdots
\end{aligned}
$$

using $\alpha^{k}=1=\beta^{k}$. Since $\left(\begin{array}{l}k \\ j\end{array}\right)$ is even for $1 \leq j<2^{i}$ and odd for $j=2^{i}$, and even for $j=2^{i}+1$, we see that all singular points of $f_{k}(x, y)$ have multiplicity $2^{i}$, except $(1,1)$ which has multiplicity $2^{i}+1$. This claim follows from

$$
\begin{aligned}
F_{0} & =\alpha^{2^{i} \ell}(\beta+1)+\beta^{2^{i} \ell}(\alpha+1)+\alpha+\beta, \\
F_{1}(x, y) & =\alpha^{2^{i} \ell} y+\beta^{2^{i} \ell} x+x+y, \\
F_{2^{i}}(x, y) & =(\beta+1) \alpha^{-2^{i}} x^{2^{i}}+(\alpha+1) \beta^{-2^{i}} y^{2^{i}}, \\
F_{2^{i}+1}(x, y) & =\alpha^{-j} x^{2^{i}} y+\beta^{-j} y^{2^{i}} x .
\end{aligned}
$$

We classify the points into three types:

(I) $P=(1,1)$.

(II) Either $\alpha=1$ or $\beta=1$ or $\alpha=\beta$.

(III) $\alpha \neq \beta$ and $\alpha \neq 1 \neq \beta$. 
Remark 3.1. If $\ell=1$ and $i>1$ the only singular point is $(1,1)$.

Defining $w(x, y):=(x+y)(x+1)(y+1)$ we note the following multiplicities on $w: m_{P}(w)=3$ if $P=(1,1)$ (Type I), $m_{P}(w)=1$ if $P$ has Type II, and $m_{P}(w)=0$ for all other singular points $P=(\alpha, \beta)$ of Type III. At long last we arrive at the multiplicities for $g_{k}(x, y)$.

\begin{tabular}{|l|c|c|c|}
\hline Type & Number of Points & $m_{P}\left(f_{k}\right)$ & $m_{P}\left(g_{k}\right)$ \\
\hline I & 1 & $2^{i}+1$ & $2^{i}-2$ \\
\hline II & $3(\ell-1)$ & $2^{i}$ & $2^{i}-1$ \\
\hline III & $(\ell-1)(\ell-2)$ & $2^{i}$ & $2^{i}$ \\
\hline
\end{tabular}

There are $3(\ell-1)$ points of multiplicity $2^{i}-1$, and so there are $(\ell-1)(\ell-2)$ singular points of multiplicity $2^{i}$ on $g_{k}(x, y)$.

Let $u$ and $v$ be projective plane curves over $\overline{G F(2)}$; we assume that $u$ and $v$ have no common component. The intersection multiplicity $I(P, u, v)$ of $u$ and $v$ at $P$ is the unique nonnegative integer satisfying and determined by the seven properties listed on pages 74-75 of [1]. For our purposes there are two important properties. One is that $I(P, u, v) \neq 0$ if and only if both $m_{P}(u)$ and $m_{P}(v)$ are $\geq 1$. Another important property is that $I(P, u, v) \geq m_{P}(u) m_{P}(v)$, with equality occurring if and only if $u$ and $v$ do not have a common tangent at $P$ (their tangent cones are disjoint).

We will use the following theorem from classical algebraic geometry, whose proof can be found in [1].

Theorem 3.3 (Bezout's Theorem). Let $u$ and $v$ be projective plane curves with no common component. Then

$$
\sum_{P} I(P, u, v)=(\operatorname{deg} u)(\operatorname{deg} v) .
$$

Our method of proving absolute irreducibility will be to assume that $g_{k}(x, y, z)$ is reducible, say $g_{k}(x, y, z)=u(x, y, z) v(x, y, z)$, and obtain a contradiction by applying Bezout's theorem to the curves $u$ and $v$. If a point $P$ has $I(P, u, v) \neq 0$, then $m_{P}\left(g_{k}\right)=m_{P}(u)+m_{P}(v) \geq 2$, and so $P$ is a singular point of $g_{k}(x, y, z)$. We have seen that the projective curves $g_{k}(x, y, z)$ have no singular points at infinity. Therefore, since the only points $P$ that give a nonzero contribution to the sum in Bezout's theorem are singular points of $g_{k}(x, y, z)$, we may just work with the affine part of $g_{k}(x, y, z)$.

\section{Homogeneous Components and Intersection Multiplicity}

The following result is clear, because we are in characteristic 2 .

Lemma 4.1. $F_{2^{i}}=(\sigma x+\tau y)^{2^{i}}$ where $\sigma=\left(\alpha^{2^{i}(\ell-1)}(\beta+1)\right)^{1 / 2^{i}}$ and $\tau=$ $\left(\beta^{2^{i}(\ell-1)}(\alpha+1)\right)^{1 / 2^{i}}$ 
Lemma 4.2. $F_{2^{i}+1}$ consists of $2^{i}+1$ different linear factors.

Proof. Consider $h(x)=F_{2^{i}+1}(x, 1)=\alpha^{2^{i}(\ell-1)} x^{2^{i}}+\beta^{2^{i}(\ell-1)} x$. If $h(x)$ has a repeated root at $a$ then $h^{\prime}(a)=0$. Consider the derivative $h^{\prime}(x)=\beta^{2^{i}(\ell-1)}$ which is never zero and therefore there are no repeated factors in $F_{2^{i}+1}$.

Next we make a crucial observation for our proofs. For the rest of this paper, we let $L=\sigma x+\tau y$, so that $F_{2^{i}}=L^{2^{i}}$. Suppose $g_{k}(x, y)=u(x, y) v(x, y)$, and suppose that the Taylor expansion at a singular point $P=(\alpha, \beta)$ is

$$
u(x+\alpha, y+\beta)=L^{r_{1}}+u_{1}, v(x+\alpha, y+\beta)=L^{r_{2}}+v_{2}
$$

where wlog $r_{1} \leq r_{2}$. Then $F_{2^{i}+1}=L^{r_{1}}\left(v_{1}+L^{r_{2}-r_{1}} u_{1}\right)$. From Lemma 4.2 we deduce that:

Lemma 4.3. With the notation of the previous paragraph,

(i) Either $r_{1}=1$ or $r_{1}=0$.

(ii) If $r_{1}=1$ then $\operatorname{gcd}\left(L, v_{1}+L^{r_{2}-r_{1}} u_{1}\right)=1$.

We next make two quick remarks to aid us in moving between $f_{k}(x, y)$ and $g_{k}(x, y)$. Recall the notation of section 3 , and suppose that $P=(\alpha, \beta) \neq(1,1)$ is a singular point of $g_{k}(x, y)$ such that $F_{2^{i}}(x, y) \neq 0$ at $P$. To apply Proposition 2 to $g_{k}$ we need to know the greatest common divisor $\left(G_{m}(x, y), G_{m+1}(x, y)\right)$ where $m=m_{P}\left(g_{t}\right)$. This can be found from $\left(F_{2^{i}}(x, y), F_{2^{i}+1}(x, y)\right)$ as follows.

Again letting $w(x, y)=(x+y)(x+1)(y+1)$, we have

$$
f_{k}(x+\alpha, y+\beta)=w(x+\alpha, y+\beta) g_{k}(x+\alpha, y+\beta),
$$

and so

$F_{2^{i}}(x, y)+F_{2^{i}+1}(x, y)+\cdots=\left(W_{0}+W_{1}(x, y)+\cdots\right)\left(G_{m}(x, y)+G_{m+1}(x, y)+\cdots\right)$.

where polynomials with subscript $i$ are 0 or homogeneous of degree $i$.

Remark 4.1. Here we assume $W_{0} \neq 0$ which is equivalent to saying that $P$ is a Type III point, and $m=2^{i}$. Multiplying out and using (2) gives

$$
\begin{aligned}
F_{2^{i}} & =W_{0} G_{2^{i}}=(\sigma x+\tau y)^{2^{i}} \\
F_{2^{i}+1} & =W_{0} G_{2^{i}+1}+W_{1} G_{2^{i}},
\end{aligned}
$$

where $\sigma^{2^{i}}=(\beta+1) \alpha^{-2^{i}}$ and $\tau^{2^{i}}=(\alpha+1) \beta^{-2^{i}}$. It follows from these equations that $\left(F_{2^{i}}, F_{2^{i}+1}\right)=\left(G_{2^{i}}, G_{2^{i}+1}\right)$.

Remark 4.2. Here we assume $W_{0}=0$ which is equivalent to saying $P$ is a Type II point, and $m=2^{i}-1$. As in Remark 4.1 we get

$$
\begin{aligned}
F_{2^{i}} & =W_{1} G_{2^{i}-1}=(\sigma x+\tau y)^{2^{i}} \\
F_{2^{i}+1} & =W_{1} G_{2^{i}}+W_{2} G_{2^{i}-1} .
\end{aligned}
$$

It is clear that (up to scalars) $W_{1}=\sigma x+\tau y$, and so $\left(F_{2^{i}}, F_{2^{i}+1}\right)=\sigma x+\tau y$ because $F_{2^{i}+1}(x, y)$ has distinct linear factors (Lemma 4.2). Hence $\left(G_{2^{i}-1}, G_{2^{i}}\right)=$ 1. 
The next result will help us to compute intersection multiplicities.

Proposition 4.4. Let $h(x, y)$ be an affine curve. Write $h(x+\alpha, y+\beta)=$ $H_{m}+H_{m+1}+\cdots$ where $P=(\alpha, \beta)$ is a point on $h(x, y)$ of multiplicity $m$. Suppose that $H_{m}$ and $H_{m+1}$ are relatively prime, and that there is only one tangent direction at $P$. If $h=u v$ is reducible, then $I(P, u, v)=0$.

Proof. See 6]

\subsection{Type I}

We upper bound the intersection multiplicity at the Type I point.

Lemma 4.5. If $g_{k}(x, y)=u(x, y) v(x, y)$ and $P=(1,1)$ then $I(P, u, v) \leq$ $\left(2^{i-1}-1\right)^{2}$.

Proof. Let $P$ be of Type I. We know that $m_{P}\left(g_{k}\right)=2^{i}-2=m_{P}(u)+m_{P}(v)$. From Lemma 4.2 we know that $F_{2^{i}+1}$ has $2^{i}+1$ different linear factors. Thus, $I(P, u, v)=m_{p}(u) m_{p}(v)$. This quantity is maximized when $m_{P}(u)=m_{P}(v)$ and in this case $m_{p}(u) m_{p}(v)=\left(2^{i-1}-1\right)^{2}$.

\section{$4.2 \quad$ Type II}

We show that intersection multiplicities at Type II points are 0 , so these points may be disregarded.

Lemma 4.6. If $g_{k}(x, y)=u(x, y) v(x, y)$ and $P=(\alpha, \beta)$ is a point of type (II) then $I(P, u, v)=0$.

Proof. There are three kinds of Type (II) point.

- If $P=(\alpha, 1)$ then $F_{2^{i}}=(\alpha+1) y^{2^{i}}$. Hence $\operatorname{gcd}\left(F_{2^{i}}, F_{2^{i}+1}\right)=y$ and therefore $\operatorname{gcd}\left(G_{2^{i}-1}, G_{2^{i}}\right)=1$ by Remark 4.2. The proof concludes using Proposition 4.4.

- If $P=(1, \beta)$ use the same argument with $\operatorname{gcd}\left(F_{2^{i}}, F_{2^{i}+1}\right)=x$.

- If $P=(\alpha, \alpha)$ use the same argument with $\operatorname{gcd}\left(F_{2^{i}}, F_{2^{i}+1}\right)=x+y$.

\subsection{Type III}

We show that there are two possibilites for the intersection multiplicity at a Type III point.

Lemma 4.7. Let $k=2^{i} \ell$ where $\ell$ is odd. If $g_{k}(x, y)=u(x, y) v(x, y)$ and $P=(\alpha, \beta)$ is a point of type (III) then either $I(P, u, v)=2^{i}$ or $I(P, u, v)=0$. 
Proof. Assume $g_{k}(x, y)=u(x, y) v(x, y)$. Since $P$ is not in $w(x, y)=(x+1)(y+$ 1) $(x+y)$ by Lemma 4.3 we know that $m_{P}(u)$ is either 1 or 0 . If $m_{P}(u)=0$ then $I(P, u, v)=0$. If $m_{P}(u)=1$ we proceed as follows.

Let $L(x, y)=\sigma x+\tau y$ and suppose we have the following Taylor expansions at $P$ :

$$
\begin{gathered}
u(x+\alpha, y+\beta)=L(x, y)+U_{2}(x, y)+\cdots \\
v(x+\alpha, y+\beta)=L(x, y)^{2^{i}-1}+V_{2^{i}}(x, y)+\cdots
\end{gathered}
$$

It follows that

$$
u(x+\alpha, y+\beta) L(x, y)^{2^{i}-2}+v(x+\alpha, y+\beta)=L(x, y)^{2^{i}-2} U_{2}(x, y)+V_{2^{i}}(x, y)+\cdots .
$$

By definition of intersection multiplicity we have

$$
I(P, u, v)=I\left(0, u(x+\alpha, y+\beta), u(x+\alpha, y+\beta) L(x, y)^{2^{i}-2}+v(x+\alpha, y+\beta)\right)
$$

so we compute the righthand side. Notice that $L(x, y) \nmid L(x, y)^{2^{i}-2} U_{2}(x, y)+$ $V_{2^{i}}(x, y)$ because $L(x, y)\left(L(x, y)^{2^{i}-2} U_{2}(x, y)+V_{2^{i}(x, y)}\right)=G_{2^{i}+1}(x, y)$ and $G_{2^{i}+1}(x, y)$ may contain $L(x, y)$ at most one time. Therefore, $u(x+\alpha, y+\beta)$ and $u(x+$ $\alpha, y+\beta) L^{2^{i}-2}+v(x+\alpha, y+\beta)$ have different tangent cones. It follows from a property of $I(P, u(x, y), v(x, y))$ that

$$
\begin{gathered}
I\left(0, u(x+\alpha, y+\beta), u(x+\alpha, y+\beta) L^{2^{i}-2}+v(x+\alpha, y+\beta)\right)= \\
m_{0}(u(x+\alpha, y+\beta)) m_{0}\left(u(x+\alpha, y+\beta) L^{2^{i}-2}+v(x+\alpha, y+\beta)\right)=2^{i} .
\end{gathered}
$$

\section{Segre Revisited.}

In this section we study the polynomials $g_{k}(x, y)$ when $k=2^{i}$ and $k=6$. First let us examine $k=2^{i}$.

$$
\begin{gathered}
f_{k}(x+1, y+1)=(x+1)^{2^{i}} y+(y+1)^{2^{i}} x+x+y \\
=x^{2^{i}} y+y^{2^{i}} x \\
=x y \prod_{\gamma \in G F\left(2^{i}\right)^{*}}(x+\gamma y) .
\end{gathered}
$$

Replace $x$ by $x+1, y$ by $y+1$, and divide by $(x+y)(x+1)(y+1)$ to get

Theorem 5.1. When $k=2^{i}$ we have the following factorization,

$$
g_{k}(x, y)=\prod_{\gamma \in G F\left(2^{i}\right) \backslash\{0,1\}}(x+\gamma y+\gamma+1) .
$$

Corollary $\mathbf{5 . 2}$ (Segre). When $k=2^{i}$ the set $D(k)$ is a hyperoval in $P G\left(2,2^{e}\right)$ if and only if $(i, e)=1$. 
Proof. We have to show that $g_{k}(x, y)$ has the necessary rational points over $G F\left(2^{e}\right)$ if and only if $(i, e)>1$.

Suppose that $i$ and $e$ are relatively prime and that there exists $a, b \in G F\left(2^{e}\right)$ with $a \neq b, a \neq 1, b \neq 1$ such that $g_{k}(a, b)=0$. By the factorization above, there exists $\gamma \in G F\left(2^{i}\right) \backslash\{0,1\}$ such that $a+\gamma b+\gamma+1=0$. But this implies $\gamma=(a+1) /(b+1) \in G F\left(2^{i}\right) \cap G F\left(2^{e}\right)=G F(2)$, a contradiction.

Conversely suppose $(i, e)>1$, and choose $a, b$ distinct in $G F\left(2^{i}\right) \cap G F\left(2^{e}\right)$ but not in $G F(2)$. Letting $\gamma=(a+1) /(b+1)$ shows that $g_{k}(a, b)=0$, again using the factorization.

We remark that $P=(1,1)$ is the only singular point in this case, and it has multiplicity $2^{i}-2$.

Next we consider $k=6$. Here

$g_{6}(x, y)=y^{4}+y^{3}(1+x)+y^{2}\left(1+x+x^{2}\right)+y\left(1+x+x^{2}+x^{3}\right)+1+x+x^{2}+x^{3}+x^{4}$.

It is easy to show that $g_{6}$ must be absolutely irreducible, or must factor over $G F(4)$ into absolutely irreducible factors. If $G F(4)=\left\{0,1, \omega, \omega^{2}\right\}$, then in fact $g_{6}=A B$ where

$$
A(x, y)=1+\omega x+x^{2}+(\omega+\omega x) y+y^{2}
$$

and its conjugate

$$
B(x, y)=1+\omega^{2} x+x^{2}+\left(\omega^{2}+\omega^{2} x\right) y+y^{2} .
$$

We have proved:

Theorem 5.3. When $k=6$ we have the factorization $g_{k}(x, y)=A(x, y) B(x, y)$, where $A(x, y)$ and $B(x, y)$ are absolutely irreducible and are given above.

Corollary 5.4 (Segre). When $k=6$ the set $D(k)$ is a hyperoval in $P G\left(2,2^{e}\right)$ if and only if $(2, e)=1$.

Proof. We have to show that $g_{6}(x, y)$ has the necessary rational points over $G F\left(2^{e}\right)$ if and only if $e$ is even.

If $e$ is even, then $g_{6}\left(\omega^{2}, \omega\right)=0$. Done.

Suppose now that $e>1$ is any odd integer. We claim that $A(x, y)$ and $B(x, y)$ have no rational points over $G F\left(2^{e}\right)$. For suppose that $A(a, b)=0$ where $a, b \in G F\left(2^{e}\right)$. Visibly we can assume $(a, b) \neq(0,0)$. Then

$$
b^{2}+b \omega+a b \omega+a^{2}+a \omega+1=0,
$$

and provided $a+b+a b \neq 0$ this implies $\omega=(a+b+1)^{2} /(a+b+a b) \in$ $G F(4) \cap G F\left(2^{e}\right)$, which is a contradiction. But if $a+b+a b=0$ then $a+b+1=$ $0 \Rightarrow a b=1 \Rightarrow 1+b^{-1}+b=0 \Rightarrow 1+b+b^{2}=0$ which is impossible. Similarly for $B(x, y)$.

Of course, these results can be proved in other ways. 


\section{The case $k \equiv 2(\bmod 4)$}

This case occurs when $k=2 \ell$.

Theorem 6.1. If $k \equiv 2(\bmod 4)$ and $k>6$ then $g_{k}(x, y)$ is absolutely irreducible.

Proof. Assume that $g_{k}(x, y)=u(x, y) v(x, y)$. Let $P=(\alpha, \beta)$ be a singular point, depending of its type we have that:

- If $P=(1,1)$ then $m_{p}\left(g_{k}\right)=0$, so $I(P, u, v)=0$.

- If $P$ is a point of type II from Lemma 4.6 we know that $I(P, u, v)=0$.

- Suppose $P=(\alpha, \beta)$ has Type III. We want to apply Proposition 4.4 to $g_{k}(x+\alpha, y+\beta)=F_{2}+F_{3}+\cdots$ where

$$
\begin{gathered}
F_{2}=\alpha^{2(\ell-1)}(\beta+1) x^{2}+\beta^{2(\ell-1)}(\alpha+1) y^{2} . \\
F_{3}=\alpha^{2(\ell-1)} x^{2} y+\beta^{2(\ell-1)} y^{2} x=x y\left(\alpha^{2(\ell-1)} x+\beta^{2(\ell-1)} y\right) .
\end{gathered}
$$

We clearly have that $\operatorname{gcd}\left(x y, F_{2}\right)=1$. If $\operatorname{gcd}\left(\alpha^{2(\ell-1)} x+\beta^{2(\ell-1)} y, F_{2}\right)=1$ then by Proposition 4.4 we have $I(P, u, v)=0$. The only way that this gcd $\neq 1$ is that $\alpha^{2(\ell-1)} x+\beta^{2(\ell-1)} y \mid F_{2}$, and this occurs if $\alpha^{4(\ell-1)}=$ $\alpha^{2(\ell-1)}(\beta+1)$ and $\beta^{4(\ell-1)}=\beta^{2(\ell-1)}(\alpha+1)$. Equivalently, $1=\alpha^{2}(\beta+1)$ and $1=\beta^{2}(\alpha+1)$. Adding both equations we get $\alpha+\beta=\alpha \beta$, multiplying then we get $1=\alpha^{2} \beta^{2}(\alpha \beta+\alpha+\beta+1)$. Substituting $\alpha+\beta=\alpha \beta$ in $1=\alpha^{2} \beta^{2}(\alpha \beta+\alpha+\beta+1)$ we get $\alpha^{2} \beta^{2}=1$ which implies $\alpha \beta=1$. So, gcd $=1$ unless $\alpha \beta=1$ and $\alpha+\beta=1$.

Suppose we have a point $P$ with $\alpha \beta=1$ and $\alpha+\beta=1$. Then $\alpha$ and $\beta$ are roots of $x^{2}+x+1$, and so they lie in $G F(4)$. This means there can be at most two such points. By Lemma 4.7, at those points $P$ we have $I(P, u, v)=0$ or 2 , and therefore $\sum_{P} I(P, u, v)=0$ or 2 or 4 . But if $k \equiv 2(\bmod 4)$ and $k>6$ then $g_{k}$ has degree at least 8 , so it is impossible that $(\operatorname{deg} u)(\operatorname{deg} v) \leq 4$. Thus we get a contradiction to Bezout's theorem.

Note that the proof fails when $k=6$, as it should, because $\sum_{P} I(P, u, v)=4$ and the two factors of $g_{6}$ have degree 2 .

\section{The case $g_{k}(x, y)$ irreducible over $\mathbb{F}_{2}$}

Here is a well known result. 
Lemma 7.1. Suppose that $p(\underline{x}) \in \mathbb{F}_{q}\left[x_{1}, \ldots, x_{n}\right]$ is of degree $t$ and is irreducible in $\mathbb{F}_{q}\left[x_{1}, \ldots, x_{n}\right]$. There there exists $r \mid t$ and an absolutely irreducible polynomial $h(\underline{x}) \in \mathbb{F}_{q^{r}}\left[x_{1}, \ldots, x_{n}\right]$ of degree $\frac{t}{r}$ such that

$$
p(\underline{x})=c \prod_{\sigma \in G} \sigma(h(\underline{x})),
$$

where $G=\operatorname{Gal}\left(\mathbb{F}_{q^{r}} / \mathbb{F}_{q}\right)$ and $c \in \mathbb{F}_{q}$. Furthermore if $p(\underline{x})$ is homogeneous, then so is $h(\underline{x})$.

Remark 7.1. Notice that if $u(x, y)=\sum a_{i, j} x^{i} y^{j}$ then $\sigma(u(x, y))=\sum \sigma\left(a_{i, j}\right) x^{i} y^{j}$ where $\sigma \in G$ is the Frobenius map (or a power of it). Therefore, $u$ and $\sigma(u)$ have the same monomials and only differ in some coefficients. This means that both $u$ and $\sigma(u)$ have the same degree.

Theorem 7.2. If $g_{k}(x, y)$ is irreducible over $\mathbb{F}_{2}$ then $g_{k}(x, y)$ is absolutely irreducible for every $k$ but $k=6$ and $k=2^{i}$.

Proof. Suppose not, then $g_{k}(x, y)=u(x, y) v(x, y)$. Using Remark 7.1 we have that $\operatorname{deg}(u)=\operatorname{deg}(v)=2^{i-1} \ell-1$. We apply Bezout's Theorem to $u$ and $v$ :

$$
\sum_{P \in \operatorname{Sin}(g)} I(P, u, v)=\operatorname{deg}(u) \operatorname{deg}(v)=\left(2^{i-1} \ell-1\right)^{2} .
$$

We can bound the left hand side as follows,

$$
\begin{aligned}
\sum_{P \in \operatorname{Sing}\left(g_{k}\right)} I(P, u, v) & =\sum_{P \in I} I(P, u, v)+\sum_{P \in I I} I(P, u, v)+\sum_{P \in I I I} I(P, u, v) \\
& \leq\left(2^{i-1}-1\right)^{2}+2^{i}(l-1)(l-2) .
\end{aligned}
$$

We have that $(8) \leq(10)$ by Bezout's Theorem, so if we prove that $(8)>(10)$ we get a contradiction. The inequality $(\underline{8})>(10)$ is

$$
\begin{gathered}
2^{2 i-2} \ell^{2}-2^{i} \ell+1>2^{i} \ell^{2}-2^{i}(3 \ell)+2^{i}+2^{2 i-2}+1 \\
\left(2^{2 i-2}-2^{i}\right) \ell^{2}+2^{i+1} \ell-2^{i}-2^{2 i-2}>0 \\
\left(2^{2 i-2}-2^{i}\right)\left(\ell^{2}-1\right)+2^{i+1}(\ell-1)>0 .
\end{gathered}
$$

The question now is when the left-hand side is positive. Clearly when $\ell=1$ it is not positive. If $\ell>1$ and $\left(2^{2 i-2}-2^{i}\right) \geq 0$ then it is clearly positive. If $\left(2^{2 i-2}-2^{i}\right)<0$ then it could be positive or negative. Clearly $\left(2^{2 i-2}-2^{i}\right)<0$ when $i=0$ or $i=1$.

If $i=0$, we have the inequality $\frac{3}{4} \ell^{2}-2 \ell+\frac{5}{4}<0$. The solutions of the equation $\frac{3}{4} \ell^{2}-2 \ell+\frac{5}{4}=0$ are 1 and $5 / 3$. Thus for $i=0$ and $\ell>1$ the polynomial $g_{k}(x, y)$ is absolutely irreducible over $\mathbb{F}_{2}$.

If $i=1$ the possible $\ell$ for which the latter equation is not positive are those solutions of the equation:

$$
-\ell^{2}+1+4 \ell-3=-\ell^{2}+4 \ell-3=0 .
$$

The possible solutions are $\ell=1$ or $\ell=3$. Hence, we conclude that except for $k=6(i=1$ and $\ell=3)$ and $k=2^{i}(\ell=1)$ the polynomial $g_{k}(x, y)$ is absolutely irreducible over $\mathbb{F}_{2}$. 


\section{Case $g_{k}(x, y)$ not irreducible over $\mathbb{F}_{2}$}

Suppose $g_{k}=f_{1} \cdots f_{r}$ is the factorization into irreducible factors over $\mathbb{F}_{2}$. Let $f_{j}=f_{j, 1} \cdots f_{j, n_{j}}$ be the factorization of $f_{j}$ into $n_{j}$ absolutely irreducible factors. Each $f_{j, s}$ has degree $\operatorname{deg}\left(f_{j}\right) / n_{j}$.

Lemma 8.1. If $P$ is a point of type III then one of the following holds:

1. $m_{P}\left(f_{j, s}\right)=0$ for all $j \in\{1, \ldots, r\}$ and $s \in\left\{1, \ldots, n_{j}\right\}$ except for a pair $\left(j_{1}, s_{1}\right)$ with $m_{P}\left(f_{j_{1}, s_{1}}\right)=2^{i}$.

2. $m_{P}\left(f_{j, s}\right)=0$ for all $j \in\{1, \ldots, r\}$ and $s \in\left\{1, \ldots, n_{j}\right\}$ except for two pair $\left(j_{1}, s_{1}\right)$ and $\left(j_{2}, s_{2}\right)$ with $m_{P}\left(f_{j_{1}, s_{1}}\right)=1$ and $m_{P}\left(f_{j_{2}, s_{2}}\right)=2^{i}-1$.

Proof. This is a consequence of Lemma 4.1 and Lemma 4.3. Consider $u=f_{a, b}$ and $v=\prod_{j \neq a, s \neq b} f_{j, s}$ from Lemma 4.3 we know that $m_{P}\left(f_{a, b}\right)$ is either 0 or 1 or $2^{i}-1$ or $2^{i}\left(\operatorname{resp} m_{p}(v)\right.$ is either $2^{i}$ or $2^{i}-1$ or 1 or 0$)$. But this is true for any pair $(a, b)$.

Clearly no two components $f_{a, b}$ and $f_{a^{\prime}, b^{\prime}}$ has multiplicity greater than or equal to $2^{i}-1$ because the total multiplicity $m_{P}\left(g_{k}\right)=2^{i}$. And there are no two components $f_{a, b}$ and $f_{a^{\prime}, b^{\prime}}$ with multiplicity equal to 1 , because then $u=f_{a, b} f_{a^{\prime}, b^{\prime}}$ has $L$ two times in the tangent cone and $v=g / u$ has $L^{2^{i-2}}$ in the tangent cone which is impossible. Hence the only possibilities are:

(i) There exists $(a, b)$ with $m_{P}\left(f_{a, b}\right)=2^{i}$, and $m_{P}\left(f_{j, s}\right)=0$ for $(j, s) \neq(a, b)$.

(ii) There exist $(a, b)$ and $\left(a^{\prime}, b^{\prime}\right)$ with $m_{P}\left(f_{a, b}\right)=1$ and $m_{P}\left(f_{a^{\prime}, b^{\prime}}\right)=2^{i}-1$, and $m_{P}\left(f_{j, s}\right)=0$ for $(j, s) \neq(a, b),(j, s) \neq\left(a^{\prime}, b^{\prime}\right)$.

Lemma 8.2. If $P$ is a point of type $I$, then for any two components $f_{a, b}$ and $f_{a^{\prime}, b^{\prime}}$ we have that $I\left(P, f_{a, b}, f_{a^{\prime}, b^{\prime}}\right)=m_{P}\left(f_{a, b}\right) m_{P}\left(f_{a^{\prime}, b^{\prime}}\right)$.

Proof. From Lemma 4.2 the tangent cones of $f_{a, b}$ and $f_{a^{\prime}, b^{\prime}}$ has no common factors.

Lemma 8.3. If $P$ is a point of type II, then for any two components $f_{a, b}$ and $f_{a^{\prime}, b^{\prime}}$ we have that $I\left(P, f_{a, b}, f_{a^{\prime}, b^{\prime}}\right)=0$.

Proof. Consider $u=f_{a, b}$ and $v=g_{m} / u$. From Lemma 4.6 we know that $I(P, u, v)=0=\sum_{(j, s) \neq(a, b)} I\left(P, u, f_{j, s}\right)$, then $I\left(P, f_{a, b}, f_{a^{\prime}, b^{\prime}}\right)=0$.

Lemma 8.4. Let $P$ is a point of type $I I I$ and $g_{k}(x, y)=\prod_{j=1}^{r} \prod_{s=1}^{n_{j}} f_{j, s}$. The intersection multiplicity $I\left(P, f_{a, b}, f_{a^{\prime}, b^{\prime}}\right)$ of any two components $f_{a, b}$ and $f_{a^{\prime}, b^{\prime}}$ is either 0 or $2^{i}$.

Proof. Consider $u=f_{a, b}$ and $v=g_{m} / u$. From Lemma 4.6 we know that either $I(P, u, v)=0=\sum_{(j, s) \neq(a, b)} I\left(P, u, f_{j, s}\right)$, then $I\left(P, f_{a, b}, f_{a^{\prime}, b^{\prime}}\right)=0$ or $I(P, u, v)=2^{i}=\sum_{(j, s) \neq(a, b)} I\left(P, u, f_{j, s}\right)$ using Lemma 8.1 we have that there exits $\left(a^{\prime}, b^{\prime}\right)$ with $I\left(P, f_{a, b}, f_{a^{\prime}, b^{\prime}}\right)=2^{i}$. 
We need some more technical results for the main theorem, which give us some upper bounds.

\section{Lemma 8.5.}

(i) If $g_{k}(x, y)$ does not have an absolutely irreducible factor over $\mathbb{F}_{2}$, then,

$$
\sum_{j=1}^{r} \operatorname{deg}\left(f_{j}\right)^{2} / n_{j}<\operatorname{deg}\left(g_{k}\right)^{2} / 2
$$

(ii)

$$
\begin{array}{r}
\sum_{j=1}^{r} \sum_{1 \leq i<s \leq n_{j}} \sum_{\substack{P \in \operatorname{Sing}\left(g_{k}\right) \\
P \neq(1,1)}} I\left(P, f_{j, i}, f_{j, s}\right)+\sum_{\substack{1 \leq j<l \leq r \\
\sum_{1 \leq i \leq n_{j}}}} \sum_{\substack{1 \leq S \leq n_{l} \\
P \neq(1,1)}} I\left(P, f_{j, i}, f_{l, s}\right) \\
\leq 2^{i}(\ell-1)(\ell-2)
\end{array}
$$

(iii)

$$
\begin{array}{r}
\left.\sum_{j=1}^{r} \sum_{1 \leq i<s \leq n_{j}} \sum_{P=(1,1)} I\left(P, f_{j, i}, f_{j, s}\right)+\sum_{\substack { 1 \leq j<l \leq r \\
\begin{subarray}{c}{1 \leq i \leq n_{j} \\
1 \leq s \leq n_{l}{ 1 \leq j < l \leq r \\
\begin{subarray} { c } { 1 \leq i \leq n _ { j } \\
1 \leq s \leq n _ { l } } }\end{subarray}} \sum_{P=(1,1)} I\left(P, f_{j, i}, f_{l, s}\right)\right) \\
\leq\left(2^{i-1}-1\right)\left(2^{i}-3\right)
\end{array}
$$

Proof. (i)

$$
\sum_{j=1}^{r} \operatorname{deg}\left(f_{j}\right)^{2} / n_{j} \leq \sum_{j=1}^{r} \operatorname{deg}\left(f_{j}\right)^{2} / 2=1 / 2\left(\operatorname{deg}\left(f_{1}\right)^{2}+\cdots+\operatorname{deg}\left(f_{r}\right)^{2}\right) \leq 1 / 2 \operatorname{deg}\left(g_{k}\right)^{2}
$$

(ii) From Lemma 8.3 we know that if $P$ is a point of type II then $I\left(P, f_{j, i}, f_{l, s}\right)=$ 0 for every $j, l \in\{1, \ldots, r\}$ and $1 \leq i \leq n_{j}, 1 \leq s \leq n_{l}$. From Lemma 8.4 we now that for each point $\mathrm{P}$ of type III there is at most two components $f_{a, b}$ and $f_{a^{\prime}, b^{\prime}}$ for which $I\left(P, f_{a, b}, f_{a^{\prime}, b^{\prime}}\right)=2^{i}$ and zero otherwise. Taking into account that there are $(\ell-1)(\ell-2)$ points of type III we get the result.

(iii) From Lemma 8.2 we have that if $P$ is a point of type $I$, then for any two components $f_{a, b}$ and $f_{a^{\prime}, b^{\prime}}$ we have $I\left(P, f_{a, b}, f_{a^{\prime}, b^{\prime}}\right)=m_{P}\left(f_{a, b}\right) m_{P}\left(f_{a^{\prime}, b^{\prime}}\right)$. Hence we have to prove the following,

$$
\begin{array}{r}
\sum_{j=1}^{r} \sum_{1 \leq i<s \leq n_{j}} m_{P}\left(f_{j, i}\right) m_{P}\left(f_{j, s}\right)+\sum_{\substack { 1 \leq j<l \leq r \\
\begin{subarray}{c}{1 \leq i \leq n_{j} \\
1 \leq s \leq n_{l}{ 1 \leq j < l \leq r \\
\begin{subarray} { c } { 1 \leq i \leq n _ { j } \\
1 \leq s \leq n _ { l } } }\end{subarray}} m_{P}\left(f_{j, i}\right) m_{P}\left(f_{j, s}\right) \\
\leq\left(2^{i-1}-1\right)\left(2^{i}-3\right) .
\end{array}
$$


Notice that the left hand side is a maximum when $m_{P}\left(f_{j, s}\right)=1$ for every $j \in\{1, \ldots, r\}, s \in\left\{1, \ldots, n_{j}\right\}$. The latter equation is

$$
\begin{gathered}
\sum_{j=1}^{r} \sum_{1 \leq i<s \leq n_{j}} m_{P}\left(f_{j, i}\right) m_{P}\left(f_{j, s}\right)+\sum_{1 \leq j<l \leq r} \sum_{\substack{1 \leq i \leq n_{j} \\
1 \leq s \leq n_{l}}} m_{P}\left(f_{j, i}\right) m_{P}\left(f_{j, s}\right) \\
\leq \sum_{j=1}^{r} \sum_{1 \leq i<s \leq n_{j}} 1+\sum_{\substack { 1 \leq j<l \leq r \\
\begin{subarray}{c}{1 \leq i \leq n_{j} \\
1 \leq s \leq n_{l}{ 1 \leq j < l \leq r \\
\begin{subarray} { c } { 1 \leq i \leq n _ { j } \\
1 \leq s \leq n _ { l } } }\end{subarray}} 1 \\
=\left(\begin{array}{c}
2^{i}-2 \\
2
\end{array}\right)=\left(2^{i}-2\right)\left(2^{i}-3\right) / 2=\left(2^{i-1}-1\right)\left(2^{i}-3\right) .
\end{gathered}
$$

Finally, here is our main result.

Theorem 8.6. $g_{k}(x, y)$ always has an absolutely irreducible factor over $\mathbb{F}_{2}$.

Proof. We apply Bezout's Theorem one more time to the product

$$
f_{1} f_{2} \ldots f_{r}=\left(f_{1,1} \ldots f_{1, n_{1}}\right)\left(f_{2,1} \ldots f_{2, n_{2}}\right) \ldots\left(f_{r, 1} \ldots f_{r, n_{r}}\right) .
$$

The sum of the intersection multiplicities can be written

$$
\sum_{j=1}^{r} \sum_{1 \leq i<s \leq n_{j}} \sum_{P \in \operatorname{Sing}\left(g_{k}\right)} I\left(P, f_{j, i}, f_{j, s}\right)+\sum_{1 \leq j<l \leq r} \sum_{\substack{1 \leq i \leq n_{j} \\ 1 \leq s \leq n_{l}}} \sum_{P \in \operatorname{Sing}\left(g_{k}\right)} I\left(P, f_{j, i}, f_{l, s}\right)
$$

where the first term is for factors within each $f_{j}$, and the second term is for cross factors between $f_{j}$ and $f_{l}$. Using Lemma 8.5, part (ii) and (iii), the previous sums can be bounded by

$$
\leq\left(2^{i-1}-1\right)\left(2^{i}-3\right)+2^{i}(l-1)(l-2) .
$$

On the other hand, the right-hand side of Bezout's Theorem is

$$
\sum_{j=1}^{r} \sum_{1 \leq i<s \leq n_{j}} \operatorname{deg}\left(f_{j, i}\right) \operatorname{deg}\left(f_{j, s}\right)+\sum_{1 \leq j<l \leq r} \sum_{\substack{1 \leq i \leq n_{j} \\ 1 \leq s \leq n_{l}}} \operatorname{deg}\left(f_{j, i}\right) \operatorname{deg}\left(f_{l, s}\right) .
$$

Since each $f_{j, s}$ has the same degree for all $s$, the first term is equal to

$$
\sum_{j=1}^{r} \operatorname{deg}\left(f_{j}\right)^{2} \frac{n_{j}-1}{2 n_{j}}=\frac{1}{2} \sum_{j=1}^{r} \operatorname{deg}\left(f_{j}\right)^{2}-\frac{1}{2} \sum_{j=1}^{r} \frac{\operatorname{deg}\left(f_{j}\right)^{2}}{n_{j}} .
$$


Note that

$$
\begin{aligned}
\left(\operatorname{deg}\left(g_{k}\right)\right)^{2} & =\left(\sum_{j=1}^{r} \operatorname{deg}\left(f_{j}\right)\right)^{2} \\
& =\sum_{j=1}^{r} \operatorname{deg}\left(f_{j}\right)^{2}+2\left(\sum_{1 \leq j<l \leq r} \operatorname{deg}\left(f_{j}\right) \operatorname{deg}\left(f_{l}\right)\right) \\
& =\sum_{j=1}^{r} \operatorname{deg}\left(f_{j}\right)^{2}+2 \sum_{1 \leq j<l \leq r}\left(\sum_{s=1}^{n_{j}} \operatorname{deg}\left(f_{j, s}\right)\right)\left(\sum_{i=1}^{n_{l}} \operatorname{deg}\left(f_{l, i}\right)\right) \\
& =\sum_{j=1}^{r} \operatorname{deg}\left(f_{j}\right)^{2}+2 \sum_{1 \leq j<l \leq r} \sum_{\substack{1 \leq i \leq n_{j} \\
1 \leq s \leq n_{l}}} \operatorname{deg}\left(f_{j, i}\right) \operatorname{deg}\left(f_{l, s}\right) .
\end{aligned}
$$

Substituting both of these into (13) shows that (13) is equal to

$$
\frac{1}{2}\left(\operatorname{deg}\left(g_{k}\right)^{2}-\sum_{j=1}^{r} \frac{\operatorname{deg}\left(f_{j}\right)^{2}}{n_{j}}\right) .
$$

Using (11) we get

$$
\frac{1}{2}\left(\operatorname{deg}\left(g_{k}\right)^{2}-\sum_{j=1}^{r} \frac{\operatorname{deg}\left(f_{j}\right)^{2}}{n_{j}}\right)>\frac{1}{2}\left(\operatorname{deg}\left(g_{k}\right)^{2}-\operatorname{deg}\left(g_{k}\right)^{2} / 2\right)=\operatorname{deg}\left(g_{k}\right)^{2} / 4 .
$$

Comparing (15) and (12), so far we have shown that Bezout's Theorem implies the following inequality:

$$
\operatorname{deg}\left(g_{k}\right)^{2} / 4 \leq\left(2^{i-1}-1\right)\left(2^{i}-3\right)+2^{i}(\ell-1)(\ell-2) .
$$

Let us now show that the opposite is true, to get a contradiction. Suppose

$$
\operatorname{deg}\left(g_{k}\right)^{2} / 4>\left(2^{i-1}-1\right)\left(2^{i}-3\right)+2^{i}(\ell-1)(\ell-2) .
$$

Then

$$
\begin{gathered}
\left(2^{2 i-2} \ell^{2}-2^{i} \ell+1\right)>\left(2^{i-1}-1\right)\left(2^{i}-3\right)+2^{i}\left(\ell^{2}-3 \ell+2\right) . \\
2^{2 i-2}\left(\ell^{2}-2\right)>2^{i} \ell^{2}-22^{i} \ell-2^{i-1} . \\
2^{2 i-2}\left(\ell^{2}-2\right)>2^{i}\left(\ell^{2}-2 \ell+1\right)-32^{i-1} . \\
2^{2 i-2}\left(\ell^{2}-2\right)>2^{i}(\ell-1)^{2}-32^{i-1} .
\end{gathered}
$$

If $\ell>1$ : the latter equation is equivalent to $2^{i-2}>\frac{(\ell-1)^{2}}{\left(\ell^{2}-2\right)}-\frac{3}{2\left(\ell^{2}-2\right)}$. One can easily see that for $\ell>1$ we have that $(\ell-1)^{2}<\left(\ell^{2}-2\right)$. Thus, $\frac{(\ell-1)^{2}}{\left(\ell^{2}-2\right)}-\frac{3}{2\left(\ell^{2}-2\right)}<$ 
1 and $2^{i-2} \geq 1$ iff $i \geq 2$. Only left to study cases $i=1$. If $i=1$ we apply Theorem 6.1.

If $\ell=1:-2^{2 i-2}>-32^{i-1} \Leftrightarrow 2^{i-2}<3 / 2$. For $i \geq 3$ this is clearly not true. So the only doubts arise for $i=1,2$. If $i=1,1 / 2<3 / 2$, and if $i=21<3 / 2$, therefore when $\ell=1$ and $i \geq 1$ we do not have a contradiction.

\section{References}

[1] W. Fulton, Algebraic Curves, Benjamin, New York, 1969.

[2] D. G. Glynn, "Two New Sequences of Ovals in Finite Desarguesian Planes of Even Order," in Combinatorial Mathematics X, Springer Lecture Notes in Mathematics 1036, Springer-Verlag, 1983, 217-229.

[3] D. G. Glynn, "A Condition for the Existence of Ovals in $P G(2, q), q$ even," Geometriae Dedicata 32 (1989), 247-252.

[4] J. W. P. Hirschfeld, Projective Geometries over Finite Fields, Clarendon Press, Oxford, 1989.

[5] J. W. P. Hirschfeld, "Ovals in Desarguesian Planes of Even Order," Ann. Mat. Pura Appl. 102 (1975), 79-89.

[6] Janwa, H.; McGuire, G.; Wilson, R. M.; Double-error-correcting cyclic codes and absolutely irreducible polynomials over GF(2).; J. Algebra 178 (1995), no. 2, 665-676.

[7] R. Lidl and H. Niederreiter, Finite Fields, Encyclopedia Math. Appl., vol.20, Addison-Wesley, Reading, MA, 1983.

[8] R. Matthews, "Permutation Properties of the Polynomials $1+x+\cdots+x^{k}$ over a Finite Field," Proc. A.M.S., 120 (1994), 47-51.

[9] C. M. O'Keefe and T. Penttila, "Hyperovals in $P G(2,16)$," European J. Combin. 12 (1991), 51-59.

[10] B. Segre, "Sui $k$-archi nei piani finiti di caratteristica 2," Revue de Math. Pures Appl. 2 (1957), 289-300.

[11] B. Segre, "Ovali e curve $\sigma$ nei piani di Galois di caratteristica due," Atti Accad. Naz. Lincei Rend. 8, 32 (1962), 785-790.

[12] B. Segre and U. Bartocci, "Ovali ed altre curve nei piani di Galois di caratteristica due," Acta Arithmetica 18, (1971), 423-449.

[13] W. M. Schmidt, Equations over Finite Fields, Springer Lecture Notes in Mathematics, 536, Springer-Verlag, 1976, 210. 